\title{
Dimensionamento e avaliação de wetland construído utilizado para tratamento de efluente doméstico em área rural: um estudo de caso
}

O uso de wetlands construídos no tratamento descentralizado de efluentes é uma forma de melhorar o saneamento das populações desfavorecidas e alternativa para as áreas rurais. O presente estudo objetivou dimensionar e avaliar o desempenho do tratamento de efluentes domésticos em área rural através do sistema tipo wetland construído de fluxo horizontal (WCFH), precedido de tanque séptico. O tanque séptico foi dimensionado e construído obedecendo a NBR $7229 / 1993$, já o WCFH, seguiu as orientações de Philippi et al. (2004), e vegetado com macrófitas da família Cannaceae. O sistema entrou em operação fevereiro de 2018. O monitoramento do sistema se deu por meio de coletas pontuais para análises físico-químicas e bacteriológicas, por um período de dois meses. A unidade apresentou eficiências de remoção de 79 \% para turbidez, 68\% para demanda bioquímica de oxigênio, 55 \% para demanda química de oxigênio, $21 \%$ de nitrogênio total Kjeldahl, 31\% para coliformes totais e 11\% para Escherichia coli. Devido ao elevado carregamento de sólidos aplicado na seção transversal do WCFH, indícios de colmatação foram observados no material filtrante após 45 dias de operação. No entanto, mesmo com este problema, o desempenho do tratamento fo satisfatório, sendo uma alternativa para o tratamento de esgoto doméstico.

\section{Dimensioning and evaluation of constructed wetland used for domestic effluent treatment in rural areas: a case study}

\begin{abstract}
The use of constructed wetlands in the decentralized treatment of domestic effluents is a way to improve the sanitation of disadvantaged populations and a clear alternative for rural areas. The present study aimed to design and evaluate the performance of domestic wastewater treatment in rural areas through a horizontal subsurface flow constructed wetland (HSSF-CW), preceded by a septic tank. The septic tank was designed and built according to NBR 7229/1993, while for HSSF $\mathrm{CW}$, it followed the guidelines of Philippi et al. (2004), and was vegetated with macrophytes of the Cannaceae family. The system went into operation in February 2018 and was monitored by specific collections for physicochemical and bacteriological analyzes, during a period of three months. The unit showed removal efficiencies of $79 \%$ for turbidity, $68 \%$ for biochemical oxygen demand, $55 \%$ for chemical oxygen demand, $21 \%$ for total Kjeldahl nitrogen, $31 \%$ for total coliforms, and $11 \%$ for Escherichia coli. Due to the high loading of solids applied in the HSSF-CW cross-section, signs of clogging were observed in the filter material after 45 days of operation. However, even with this problem, the performance of the treatment was satisfactory, being an alternative for the treatment of domestic sewage.
\end{abstract}

Keywords: Constructed wetlands; Basic sanitation; Decentralized treatment

Topic: Engenharia Sanitária

Reviewed anonymously in the process of blind peer.
Received: 04/06/2021

Approved: $26 / 06 / 2021$
Daniela Sartor (iD)

Universidade Federal de Santa Maria, Brasil http://lattes.cnpq.br/0472179105617192 http://orcid.org/0000-0003-3130-2995

daniela sartor@hotmail.com

\section{Janieli Aparecida Minski da Motta}

Universidade Federal de Santa Maria, Brasil

janieli_motta@hotmail.com

Raphael Corrêa Medeiros (iD)

Universidade Federal de Santa Maria, Brasi

http://lattes.cnpq.br/2977594460581447

http://orcid.org/0000-0002-7090-1731

medeiroscg@yahoo.com.br

\section{Samara Terezinha Decezaro (10) \\ Universidade Federal de Santa Maria, Brasil \\ http://lattes.cnpq.br/4958707293395412 \\ http://orcid.org/0000-0002-0812-7749 \\ samara.decezaro@ufsm.br}

Referencing this:

SARTOR, D.; MOTTA, J. A. M.; MEDEIROS, R. C.; DECEZARO, S. T.. Dimensionamento e avaliação de wetland construído utilizado para tratamento de efluente doméstico em área rural: um estudo de caso. Revista Ibero Americana de Ciências Ambientais, v.12, n.6, p.288296, 2021. DOI: http://doi.org/10.6008/CBPC21796858.2021.006.0024 


\section{INTRODUÇÃO}

A Lei Federal 11.445, de 2007 (BRASIL, 2007), considera que o saneamento básico é um conjunto de serviços, infraestruturas e instalações operacionais de abastecimento de água potável, esgotamento sanitário, limpeza urbana e manejo de resíduos sólidos e drenagem e manejo de águas pluviais urbanas. Como forma de alcançar um ambiente capaz de proporcionar condições favoráveis à saúde da população, evitando assim a ocorrência de doenças transmitidas através do meio ambiente, objetiva-se alcançar o saneamento ambiental.

Aproximadamente $16 \%$ da população brasileira residem na zona rural. Quando comparada à população urbana, está vivendo em situação precária no acesso a saneamento ambiental, tornando assim um determinante social da saúde. Segundo o 24 Diagnóstico dos Serviços de Água e Esgotos, em 2018, 46,3\% dos esgotos gerados foram tratados (SNIS, 2019). Porém, estima-se que são 23 milhões de pessoas residam na zona rural sem coleta ou tratamento de esgoto (COSTA et al., 2019).

Os wetlands construídos (WCs) vêm sendo utilizados como forma descentralizada no tratamento de efluentes, normalmente, precedidos de tanque séptico, e demonstram grande potencial de aplicação nas áreas rurais. Apresentam custos de operação e manutenção relativamente baixos, simplicidade e flexibilidade operacional e índices de remoção de poluentes considerados eficientes; porém apresentam limitações, como a dependência do meio ambiente e grande área demandada, além de estar sujeito a entupimento do espaço poroso (PERONDI et al., 2020; MATOS et al., 2018; DOTRO et al., 2017).

O termo wetland caracteriza os ecossistemas que ficam inundados total ou parcialmente. Esses sistemas são conhecidos na forma natural como: banhados, terras úmidas ou pântanos. Já na forma construída, pode-se obter controle sobre ele, que na literatura científica, podem-se encontrar além de wetlands, outras denominações, como: zona de raízes, jardins filtrantes, sistemas alagados construídos, entre outras. Neles, ocorrem simultaneamente processos de ordem química, física e bioquímicas, abrangendo a vegetação, meio suporte bem como a biota aderida (EPA, 2000; SEZERINO et al., 2015).

Os WCs são uma tecnologia em desenvolvimento no Brasil, que apesar de ainda não estar normatizada pela $A B N T$, apresenta grande potencial para aplicação, pois o país apresenta vantagem favorável em relação à condição climática e disponibilidade territorial, oferecida pela maior parte das regiões. Além do mais, o efluente tratado pode ser reutilizado, bem como as macrófitas. O sistema é naturalmente integrado ao meio ambiente, conservando os aspectos paisagísticos do meio rural.

Neste contexto, o presente trabalho teve como objetivo dimensionar e avaliar o desempenho de um wetland construído de fluxo horizontal (WCFH) precedido de tanque séptico no tratamento de efluentes domésticos em área rural.

\section{MATERIAIS E MÉTODOS}

O sistema de tratamento de efluentes foi estudado em uma propriedade rural (latitude $27^{\circ}-48^{\prime} 30.74^{\prime \prime}$ sul e longitude 541' $7.33^{\prime \prime}$ oeste), composta por duas residências com 8 moradores no total, situado em 
Quarainzinho, no município de Três de Maio, localizado na região noroeste do Rio Grande do Sul.

A geração de efluentes nas residências é resultante dos diferentes usos, como o uso da água para atividades domésticas, limpeza pessoal, atividades de pequena agroindústria familiar (feitio de bolos, queijo e doces), e foi estimada, através da contribuição diária de despejos presente na NBR 13969/1997, em aproximadamente $1.280 \mathrm{~L} \mathrm{~d}^{-1}$.

A partir da necessidade de melhoramento do saneamento básico da propriedade, foi dimensionado e instalado para efetuar o tratamento dos efluentes gerados, conforme a Figura 1, um tanque séptico seguido de um WCFH, com saturação constante, alimentação abaixo da superfície e com macrófitas emergentes (FONDER et al., 2013).

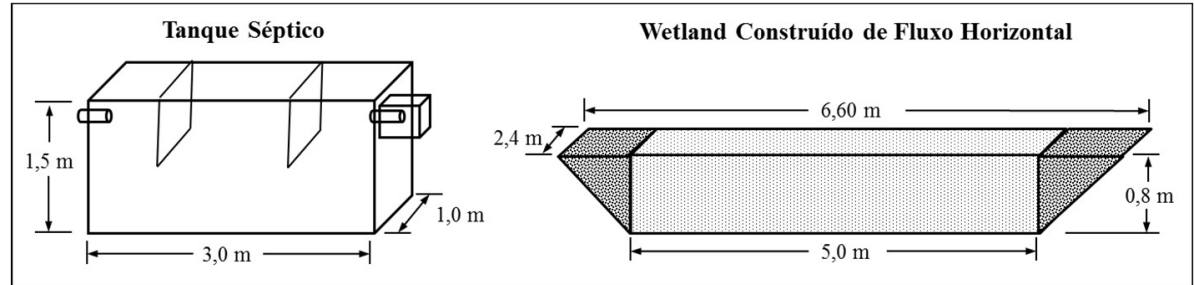

Figura 1: Diagrama esquemático das unidades do sistema de tratamento de esgoto rural, com algumas medidas principais, do tipo WC precedida por tanque séptico.

O tanque séptico foi projetado e construído atendendo às normativas impostas na NBR 7229 (ABNT, 1993). Para definir o volume útil do tanque séptico, utilizou-se como taxa de acumulação total de lodo (K) o valor de 225 dias, com intervalo entre limpezas de 5 anos e temperatura do mês mais frio, entre 10 e 20 ㅇ․ O dimensionamento resultou na escolha de um tanque prismático retangular de câmara única, com volume de $4.500 \mathrm{~L}$, construído em alvenaria de tijolo maciço, revestidos internamente com $1,5 \mathrm{~cm}$ de argamassa de cimento e areia no traço 1:3, para impermeabilização. Na saída do tanque séptico, foi construída, para fins de inspeção e coleta, uma caixa impermeável de alvenaria nas dimensões de 0,6 × 0,6 x 0,6 metros.

O WCFH foi dimensionado conforme metodologia de Philippi et al. (2004), sendo adotado o valor de $400 \mathrm{mg} \cdot \mathrm{L}^{-1}$ de $\mathrm{DBO}_{5}$ no efluente bruto, de acordo com o indicado por Von Sperling (2017). O WCFH foi construído em solo escavado com taludes em 45e e impermeabilizado com geomembrana com espessura de 800 micras. Por conta do talude, a área superior resultante foi de $26,4 \mathrm{~m}^{2}$. 0 efluente a ser tratado, era disposto no primeiro metro do leito, denominada zona de entrada, por meio de um tubo de esgoto, perfurado ao longo da extensão, envolto por pedra brita número 1 . Assim, o efluente vai penetrando vagarosamente, em fluxo horizontal, através do material filtrante composto de areia média, até atingir a zona de saída, composta também por pedra brita número 1. Na zona de saída, o esgoto tratado é coletado por tubo de esgoto perfurado. O nível de efluente dentro do WCFH foi mantido em torno de $50 \mathrm{~cm}$.

Após o WCFH, foi construída em alvenaria, uma caixa destinada para inspeção, coleta de amostras e controladora de nível do WCFH. O material filtrante foi vegetado com macrófitas adultas com brotação no rizoma da família Cannaceae, na densidade de oito plantas por metro quadrado. As macrófitas foram coletadas em seu ambiente natural, e tiveram as raízes lavadas e folhas cortadas para, então, serem plantadas sobre o material filtrante do WCFH. 
O monitoramento do experimento compreendeu o plantio e replantio das macrófitas, quando necessário, coleta de amostras de efluentes e respectivas análises laboratoriais. As análises físico-químicas e microbiológicas do efluente foram realizadas em dois pontos de coleta: após o tanque séptico e após o WCFH, seguindo as metodologias de acordo com a Tabela 1.

Tabela 1: Metodologias utilizadas dos parâmetros físico-químicas e microbiológicos analisados.

\begin{tabular}{lll} 
Parâmetros & Metodologia & Unidade \\
\hline Coliformes totais e Escherichia coli & Tubos múltiplos e meio de cultura Colilert ${ }^{\circledR}$. & $\mathrm{NMP} / 100 \mathrm{~mL}$ \\
\hline DBO $_{5,20}$ & Método de Winkler (APHA et al., 2012). & $\mathrm{mg}$. L- $^{-1}$ \\
\hline DQO & Colorimétrico por refluxo fechado (APHA et al., 2012). & $\mathrm{mg}^{-1}-^{-1}$ \\
\hline Nitrogênio total Kjeldahl (NTK) & Macro-Kjeldahl - (APHA et al., 2012) & $\mathrm{mg.L-}^{-1}$ \\
\hline pH & Método potenciométrico & - \\
\hline Sólidos totais & Método gravimétrico (APHA et al., 2012) & $\mathrm{mg.L-1}^{-1}$ \\
\hline Turbidez & Método nefelométrico & UNT \\
\hline Temperatura & Termômetro de vidro & oC \\
\hline
\end{tabular}

A areia média utilizada no leito filtrante apresentou como índice granulométrico em análise laboral (NBR 7181/1984 - Análise Granulométrica), diâmetro efetivo $\left(d_{10}\right)$ de $0,34 \mathrm{~mm}$; diâmetro a $60 \%\left(d_{60}\right)$ de 0,50 mm e coeficiente de uniformidade $(\mathrm{Cu})$ 1,47. Esses valores são considerados aceitáveis para emprego em wetlands construídos, visto que: $\mathrm{d}_{10}$ superior ou igual a $0,20 \mathrm{~mm}$; coeficiente de uniformidade menor ou igual a 5 unidades (PHILIPPI et al., 2004).

\section{RESULTADOS E DISCUSSÃO}

A tabela 2 apresenta os resultados de quase 100 dias de monitoramento do WCFH.

Tabela 2: Valores médios ( \pm desvio padrão) dos parâmetros de controle e eficiência no sistema WC, precedido por tanque séptico.

\begin{tabular}{|c|c|c|c|}
\hline Parâmetros & Efluente após tanque séptico & Efluente após WCFH & Eficiência de remoção WCFH (\%) \\
\hline Temperatura (으) & $22 \pm 3$ & $22 \pm 3$ & \\
\hline pH & $5,5 \pm 0,3$ & $6,3 \pm 0,3$ & ------ \\
\hline Turbidez (UNT) & $289 \pm 57$ & $61 \pm 45$ & 79 \\
\hline DBO (mg. $\mathrm{L}^{-1}$ ) & $494 \pm 97$ & $156 \pm 544$ & 68 \\
\hline DQO (mg.t-1) & $1337 \pm 618$ & $596 \pm 318$ & 55 \\
\hline Sólidos Totais (mg. $\mathrm{L}^{-1}$ ) & $1258 \pm 420$ & $1167 \pm 336$ & 7 \\
\hline NTK (mg. $\mathrm{L}^{-1}$ ) & $60 \pm 58$ & $47 \pm 50$ & 21 \\
\hline Coliformes totais (NMP/100mL) & $1,12 \times 10^{7}$ & $7,72 \times 10^{6}$ & 31 \\
\hline Escherichia coli (NMP/100mL) & $5,57 \times 10^{5}$ & $4,95 \times 10^{5}$ & 11 \\
\hline
\end{tabular}

Observa-se pH levemente ácido do efluente do tanque séptico, sendo reflexo da degradação da matéria orgânica e produção de ácidos voláteis, a partir de processos anaeróbios (VON SPERLING, 2017). Já no WCFH, o pH encontrado foi de 6,30, indicando condições ideias para a sobrevivência de bactérias responsáveis pela degradação orgânica. Porém, ainda fora da faixa ótima para a ocorrência de nitrificação, varia entre 7,5 e 8,6. O comportamento do $\mathrm{pH}$ do afluente e efluente ao $\mathrm{WCFH}$, no decorrer do monitoramento, pode ser visualizado na Figura 2.

A relação DQO/DBO foi de 0,85 no tanque séptico e de 1,21 após o WCFH, apontando redução na fração biodegradável. No entanto, segundo Von Sperling (2017), quando menor que 2,5, a fração biodegradável é elevada. Nota-se, portanto, que mesmo após passar pelas duas unidades de tratamento, a 
fração biodegradável no efluente permaneceu elevada, sendo ainda necessário um pós-tratamento do efluente do WCFH, a fim de evitar impactos no ambiente onde é lançado. As remoções de matéria orgânica e nitrogênio estão expostas na Figura 3.

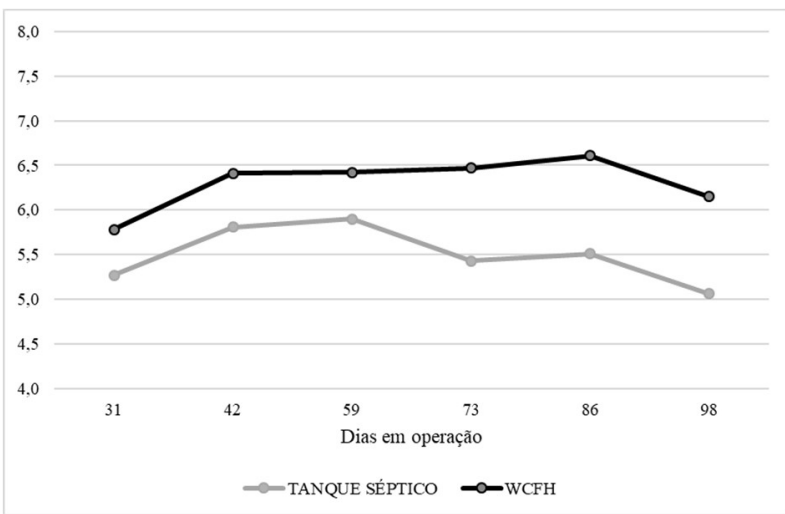

Figura 2: Comportamento do $\mathrm{pH}$ ao longo do tempo de operação do WCFH.

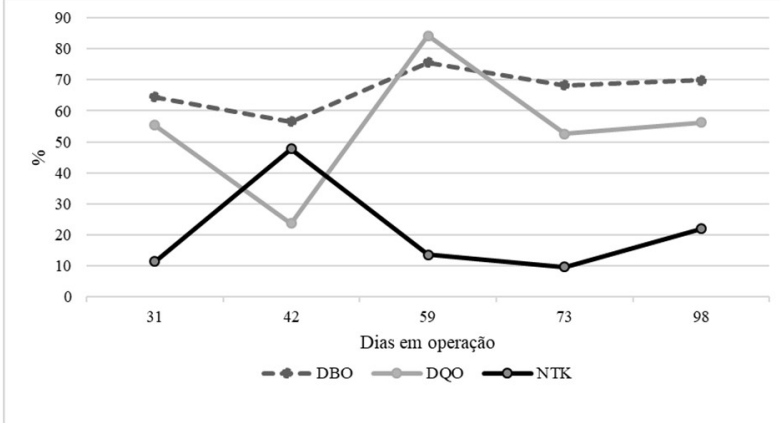

Figura 3: Remoção de DBO, DQO e NTK em WCFH implantado em área rural.

Lourenço et al. (2018) utilizando WCFH como pós-tratamento de esgoto doméstico em filtro anaeróbio em análise por seis meses, obtiveram como eficiência média na remoção de DQO de $62 \%$ e DBO 64\%; bastante próximas ao presente estudo.

A DQO obteve valores de redução média em $55 \%$, no entanto, considerando que a avaliação foi realizada no início de operação, período necessário para adaptação das plantas e micro-organismos, a eficiência do WCFH tende a aumentar com o passar do tempo para remoção de matéria orgânica, conforme conclui Shahamat et al. (2018).

Observa-se redução média em termos de DBO de $68 \%$, ficando abaixo do esperado pela equação utilizada no dimensionamento, a qual considerou remoção de $86 \%$. Isso pode ser devido à elevada carga orgânica presente no afluente do tanque séptico, que recebeu juntamente com o esgoto doméstico, soro de leite. Alguns autores destacaram que o soro do leite apresenta DBO que pode variar de 27.000 a 60.000 mg.L 1. dessa forma, o valor adotado de $400 \mathrm{mg} . \mathrm{L}^{-1}$ de DBO, do efluente sanitário para o dimensionamento do WC, foi subdimensionado, pois a média de DBO na saída do tanque séptico apresentou concentração de 500 mg.L1 .

A Resolução CONAMA no 430 de 2011 (CONAMA, 2011), considera no artigo 21, remoção mínima de $\mathrm{DBO}_{5,20}$ em $60 \%$, desta forma o WCFC atende de forma satisfatória às condições e aos padrões de lançamento para efluentes de sistemas de tratamento de esgotos sanitários no que se refere à DBO.

Praticamente todo o ciclo do nitrogênio é ativo nos WCFH como os processos de: mineralização (amonificação), volatilização do amoníaco, nitrificação, desnitrificação, absorção de plantas e microrganismos, fixação de nitrogênio, redução de nitrato, oxidação anaeróbica de amoníaco, adsorção, dessorção e lixiviação, bem como por rotas de remoção de nitrogênio recentemente descobertas, as quais dependem exclusivamente do metabolismo microbiológico, como a nitrificação parcial-desnitrificação, processo Anammox e Canon (PHILIPPI et al., 2004; VYMAZAL, 2007; HOFFMANN et al., 2011; SAEED et al., 2012). 
A concentração de DBO, normalmente cai rapidamente após a maturação do sistema, é necessário entre duas semanas até seis meses para o desenvolvimento da nitrificação (EPA, 1978). Neste trabalho, como pode ser analisado na Figura 3, não ocorreu nitrificação nem a estabilização do tratamento durante o acompanhamento.

A eficiência média de remoção de NTK foi de $21 \%$. No entanto, próxima aos resultados que Costa et al. (2018) obtiveram, após análises semanais, durante quatro anos, de dois WCFH precedidos de um sistema anaeróbico: no sistema não plantado, 15\%; já na unidade que continha macrófitas, a remoção média foi de $30 \%$.

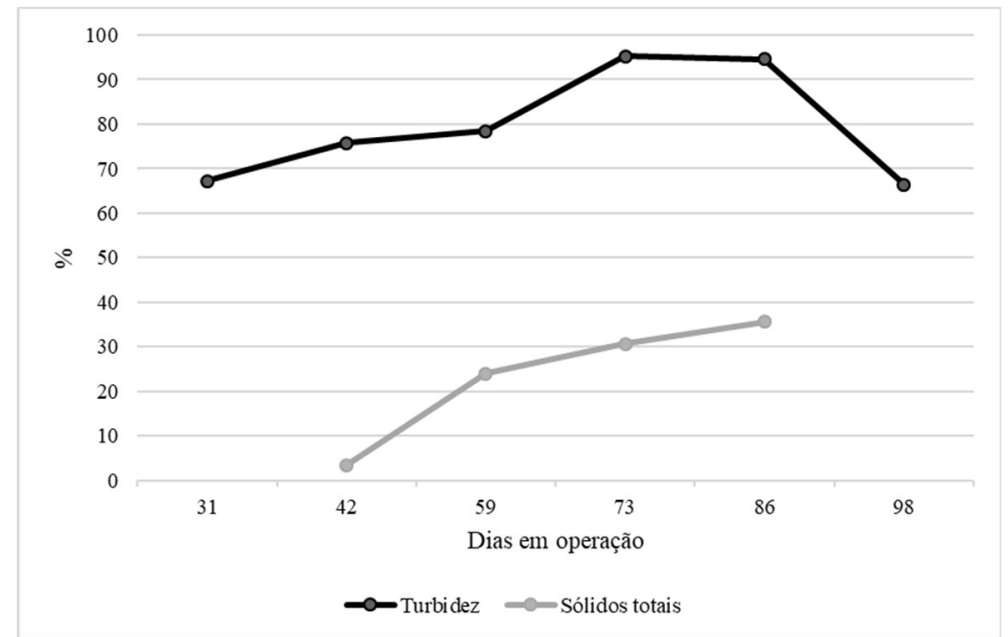

Figura 4: Remoção de turbidez e sólidos totais em WCFH implantado em área rural.

Verifica-se boa remoção da turbidez, média de redução de 79 \% (Figura 4), o que pode ser devido ao tempo de detenção hidráulica de 3 dias. A turbidez pode estar sendo removida pelos processos de: filtração, sedimentação, assimilação de plantas e/ou metabolismo microbiano. A remoção de turbidez apresentou características de crescente remoção, exceto no 98 dia de operação do WCFH, em que devido a ocorrências pluviométricas, pode ter havido lixiviação no sistema, dias antes da coleta.

Shahamat et al. (2018), em estudo com duas plantas locais diferentes empregadas em WCFH distintos, obtiveram como resultados de eficiência para a turbidez de 79,8\% e 71,5\%, para a DBO 93,7\% e 79,1\%, já para a DQO 82,6\% e 68,8\%. Resultados próximos ao do presente trabalho. A média de sólidos totais encontrados após o WCFH foi de 1167 mg.L-1 (Figura 4). Porém observou-se crescente remoção com o tempo de operação, com até $40 \%$, na última análise realizada.

Segundo a edição especial do boletim Wetlands Brasil (VON SPERLING et al., 2018), é indicado como taxa de aplicação orgânica superficial máxima, valor entre 6 e $15 \mathrm{~g} \mathrm{DBO} \cdot \mathrm{m}^{-2} \cdot \mathrm{d}^{-1}$, porém foi encontrada a taxa de $76 \mathrm{~g} \mathrm{DBO} \cdot \mathrm{m}^{-2} \cdot \mathrm{d}^{-1}$. Já a taxa de aplicação orgânica máxima na seção transversal, o indicado é de até $250 \mathrm{~g}$ DBO. $\mathrm{m}^{-2} \cdot \mathrm{d}^{-1}$, porém no trabalho aplicou-se $1670 \mathrm{~g}$ DBO. $\mathrm{m}^{-2} \cdot \mathrm{d}^{-1}$.

Esses valores aplicados acima do recomendado, devido à mistura do esgoto doméstico com o soro de leite, podem ter afetado o sistema. Observou-se, nos horários de maior uso de água nas residências, escoamento superficial na entrada do WCFH, após 16 dias de operação; e após 45 dias, o fenômeno pode ser observado ao longo do WCFH.

O processo de entupimento é inevitável, entretanto Vymazal (2018) destaca que o processo pode ser 
bastante desacelerado com o uso de materiais de filtragem com porosidade adequada, manutenção, cargas adequadas de orgânicos e sólidos em suspensão. Cargas orgânicas e de sólidos elevadas devem ter levado à colmatação do material filtrante da área em estudo. Além disso, outros fatores podem ter influenciado, como por exemplo, a compactação da areia durante a montagem do sistema e plantio/replantio.

Devido à presença concomitante de efluente de laticínio, a taxa de aplicação orgânica foi 5 vezes superior ao recomendado por Philippi et al. (2004), representando $65 \mathrm{~g} \mathrm{DQO} . \mathrm{m}^{-2} \cdot \mathrm{d}^{-1}$. Trein et al. (2015) notaram que, após pré-tratamento ineficiente, houve carreamento de elementos sólidos e orgânicos para o wetland, com $13 \mathrm{~g} \mathrm{SS} \cdot \mathrm{m}^{-2} \cdot \mathrm{d}^{-1}$ e $87 \mathrm{~g}$ DQO. $\mathrm{m}^{-2} \cdot \mathrm{d}^{-1}$, notando assim elevado escoamento superficial desencadeado pela colmatação do material filtrante.

O escoamento superficial pode ter dificultado os processos aeróbios de degradação, ocorrendo assim, a redução da DBO em anaerobiose, que é mais lenta que a aeróbica. A excessiva quantidade de matéria orgânica pode provocar, em curto espaço de tempo, a colmatação do material filtrante, ocasionando a diminuição da transferência de oxigênio, causando mau desempenho até colapso do sistema. Alguns autores, como Platzer et al. (1997), recomendam que tenham limite máximo de carregamento no sistema de $25 \mathrm{~g}$ DQO. $\mathrm{m}^{-2} \cdot \mathrm{d}^{-1}$.

O escoamento do efluente dentro do material filtrante é de baixa velocidade, favorecendo a sedimentação e filtração de sólidos suspensos e sua adsorção ao substrato poroso. Com o crescimento e reprodução do biofilme, os sólidos dissolvidos são "convertidos" a suspensos, levando à formação de subprodutos e exsudatos pela mineralização sofrida com o metabolismo microbiano (MATOS, 2015).

Estes mecanismos, ao longo do tempo, acumulam sólidos nos espaços porosos presentes no material filtrante, ocasionando bloqueio interno com a diminuição da porosidade, processo conhecido como colmatação, proporcionando baixa condutividade hidráulica no meio, fazendo com que o efluente se movimente por caminhos com facilidade de passagem, alterando o sistema. $O$ escoamento de subsuperficial passa a ser superficial, principalmente nos trechos iniciais do WC (MATOS, 2015).

Desta forma, o tempo de detenção hidráulica do efluente é diminuído, prejudicando a eficiência nas remoções física, química e biológica poluentes, bem como se criam condições de anaerobiose, diminuindo a efetividade do tratamento e provocando potencial geração de maus odores e proliferação de insetos (MATOS, 2015).

As remoções de coliforme totais e Escherichia coli em alguns momentos foi de uma unidade logarítmica (90\%). Mas, em média, foi inferior ao apresentado por Maiga et al. (2017), para coliformes totais e Nivala et al. (2019), em relação à Escherichia coli. No entanto, os trabalhos de ambos os autores relatam período de operação e monitoramento maior.

\section{CONCLUSÕES}

O WCFH apresentou boa eficiência de turbidez, bem como da matéria orgânica carbonácea: $\mathrm{DBO}_{5,20}$ e DQO. Em razão disso, conseguiu atender às condições e aos padrões de lançamento de efluentes de sistemas de tratamento de esgotos sanitários, de acordo com o estabelecido pela CONAMA no 430 de 2011. 
No entanto, deve-se atentar à taxa de aplicação orgânica máxima, para não sobrecarregar o sistema de tratamento. Mas, apesar da ocorrência de colmatação, a utilização de WCFH é uma alternativa eficiente para o tratamento de efluente doméstico em área rural.

\section{REFERÊNCIAS}

ABNT. Associação Brasileira de Normas Técnicas. NBR 13969: Tanques sépticos: Unidades de tratamento complementar e disposição final dos efluentes líquidos: Projeto, construção e operação. Rio de Janeiro: ABNT, 1997.

ABNT. Associação Brasileira de Normas Técnicas. NBR 7229: Projeto, construção e operação de sistemas de tanques sépticos. Rio de Janeiro: ABNT, 1993

BRASIL. Lei no 11.445, de 5 de janeiro de 2007. Brasília: DOU, 2007.

CONAMA. Conselho Nacional do Meio Ambiente. Resolução no 430, de 13 de maio de 2011. Brasília: CONAMA, 2011.

COSTA, J. F.; PAOLI, A. O.; VON SPERLING, M.; SEIDL, M.. Avaliação do desempenho de sistemas alagados construídos de escoamento horizontal subsuperficial tratando efluente de reator UASB, com base em quatro anos de monitoramento. Engenharia Sanitária e Ambiental, v.23, n.1, p.191-200, 2018. DOI: http://doi.org/10.1590/s14134152201890370

COSTA, R.; MATOS, M.; MATOS, A.; VON SPERLING, M. Comportamento hidrodinâmico de sistemas alagados construídos de escoamento horizontal subsuperficial de diferentes proporções geométricas e tempos de operação. Engenharia Sanitária e Ambiental, v.24, p.83-91, 2019. DOI: http://doi.org/10.1590/s1413-41522019175103

DOTRO, G.; LANGERGRABER, G.; MOLLE, P.; NIVALA, J.; PUIGAGUT, J.; STEIN, O.; VON SPERLING, M.. Treatment wetlands, biological wastewater treatment series. 2017.

EPA. Environmental Protection Agency. EPA 625-R-99-010: US EPA ORD. Manual Constructed Wetlands for Municipal Wastewater Treatment. Cicinnati, 2000.

EPA. Environmental Protetion Agency. EPA 625/1-08-012: Office of Research and Development, Municipal Environmental Research Laboratory, Design manual- Onsite wastewater treatment and disposal systems. Cincinnati, 1978.

FONDER, N.; HEADLEY, T.. The taxonomy of treatment wetlands: A proposed classification and nomenclature system. Ecological Engineering, v.51, p.203-211, 2013. DOI: http://dx.doi.org/10.1016/i.ecoleng.2012.12.011

HOFFMANN, H.; PLATZER, C.; WINKER, M.; VON MUENCH, $E$.. Technology review of constructed wetlands subsurface flow. Constructed wetlands for greywater and domestic wastewater treatment in developing countries. Germany: Deutsche Gesellschaft für Technische Zusammenarbeit $\mathrm{GmbH}$ (GTZ), 2011.

LOURENÇO, L. S.; RODRIGUES, E. B.; MOREIRA, M. A.; SKORONSKI, E.. Remoção de matéria orgânica e nutrientes de esgoto doméstico por wetland horizontal de fluxo subsuperficial na estação de tratamento de aparecida Campos Novos, SC. Revista Brasileira de Agropecuária Sustentável, v.8, n.1, 2018. DOI: http://doi.org/10.21206/rbas.v8i1.483

MAIGA, Y.; VON SPERLING, M.; MIHELCIC, J.. Constructed Wetlands. In: ROSE, J. B.; JIMÉNEZ-CISNEROS, B.. Global Water Pathogens Project. In: HAAS, C.; MIHELCIC, J.; VERBYLA, M.. Part 4. Management of Risk from excreta and Wastewater. Michigan State University, E. Lansing: UNESCO. 2017. p.1-17. Dol: http://doi.org/10.14321/waterpathogens.66

MATOS, M. P.. Colmatação em sistemas alagados construídos de escoamento horizontal subsuperficial: principais fatores e métodos de identificação em unidades plantadas e não plantadas. Tese (Doutorado em Saneamento, Meio Ambiente e Recursos Hídricos) Universidade Federal de Minas Gerais, Belo Horizonte, 2015.

MATOS, M. P.; VON SPERLING, M.; MATOS, A. T.. Clogging in horizontal subsurface flow constructed wetlands: influencing factors, research methods and remediation techniques. Rev Environ Sci Biotechnol, v.17, p.87-107, 2018. DOI: https://doi.org/10.1007/s11157-018-9458-1

NIVALA, J.; BOOG, J.; HEADLEY, T.; AUBRON, T.; WALLACE, S.; BRIX, H.; MOTHES, S.; MANFRED, A.; MÜLLER, R.. Side-byside comparison of 15 pilot-scale conventional and intensified subsurface flow wetlands for treatment of domestic wastewater. Science of The Total Environment, v.658, p.1500-1513, 2019. DOI: http://doi.org/10.1016/j.scitotenv.2018.12.165

PERONDI, T.; WOLFF, D. B.; DECEZARO, S. T.; ARAÚJO, R. K.. Wetlands construídos para o tratamento de esgoto doméstico: uma análise comparativa do custo do ciclo de vida. Ambiente Construído, Porto Alegre, v.20, n.2, p.175189, abr./jun. 2020. DOI: http://doi.org/10.1590/s167886212020000200394

PHILIPPI, L. S.; SEZERINO, P. H.. Aplicação de sistemas tipo wetlands no tratamento de águas residuárias: utilização de filtros plantados com macrófitas. Florianópolis: Autor, 2004.

PLATZER, C.; MAUCH, K.. Soil clogging in vertical flow reed beds - mechanisms, parameters, consequences and. solutions? Water Science and Technology, v.35, n.5, p.175181, 1997. DOI: http://doi.org/10.1016/S02731223(97)00066-8

SAEED, T.; SUN, G.. A review on nitrogen and organics removal mechanisms in subsurface flow constructed wetlands: Dependency on environmental parameters, operating conditions and supporting media. Journal of Environmental Management, v.112, p.429-448, 2012. DOI: http://doi.org/10.1016/j.jenvman.2012.08.011 
SALATI, E.; SALATI FILHO, E.; SALATI, E.. Utilização de sistemas de wetlands construídas para tratamento de águas. Piracicaba: Instituto Terramax, 2009.

SHAHAMAT, Y. D.; ASGHARNIA, H.; KALANKESH, L. R.; HOSANPOUR, M.. Data on wastewater treatment plant by using wetland method, Babol, Iran. Data In Brief, v.16, p.1056-1061, 2018. DOI:

http://doi.org/10.1016/i.dib.2017.12.034

SNIS. Sistema Nacional de Informações sobre Saneamento. 24 Diagnóstico dos Serviços de Água e Esgotos - 2018. Brasília: SNIS, 2019.

TREIN, C. M.; PELISSARI, C.; HOFFMANN, H.; PLATZER, C. J.; SEZERINO, P. H.. Tratamento descentralizado de esgotos de empreendimentos comercial e residencial empregando a ecotecnologia dos wetlands construídos. Ambiente Construído, v.15, n.4, p.351-367, 2015. DOI: http://dx.doi.org/10.1590/s1678-86212015000400055

VON SPERLING, M.. Introdução à qualidade das águas e ao tratamento de esgotos. 4 ed. Belo Horizonte: UFMG, 2017.

VON SPERLING, M.; SEZERINO, P. H.. Dimensionamento de wetlands construídos no Brasil. Boletim Wetlands Brasil, p.65, 2018.

VYMAZAL, J.. Does clogging affect long-term removal of organics and suspended solids in gravel-based horizontal subsurface flow constructed wetlands? Chemical Engineering Journal, v.331, p.663-674, 2018. DOI: http://doi.org/10.1016/i.cej.2017.09.048

VYMAZAL, J.. Removal of nutrients in various types of constructed wetlands. Science of The Total Environment, v.380, n.1-3, p.48-65, 2007. DOI:

http://doi.org/10.1016/j.scitotenv.2006.09.014

A CBPC - Companhia Brasileira de Produção Científica (CNPJ: 11.221.422/0001-03) detém os direitos materiais desta publicação. Os direitos referem-se à publicação do trabalho em qualquer parte do mundo, incluindo os direitos às renovações, expansões e disseminações da contribuição, bem como outros direitos subsidiários. Todos os trabalhos publicados eletronicamente poderão posteriormente ser publicados em coletâneas impressas sob coordenação da Sustenere Publishing, da Companhia Brasileira de Produção Científica e seus parceiros autorizados. Os (as) autores (as) preservam os direitos autorais, mas não têm permissão para a publicação da contribuição em outro meio, impresso ou digital, em português ou em tradução. 